\title{
POLYMORPHISMS IN TNFA AND IL10 GENE PROMOTERS AND RISK OF RHEUMATOID ARTHRITIS IN BULGARIAN POPULATION
}

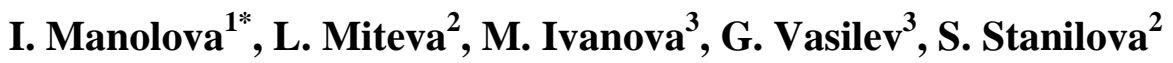 \\ ${ }^{1}$ Department of Health Care, Medical Faculty, Trakia University, Stara Zagora, Bulgaria \\ ${ }^{2}$ Department of Molecular Biology, Immunology and Medical Genetics, Medical Faculty, \\ Trakia University, Stara Zagora, Bulgaria \\ ${ }^{3}$ Clinic of Rheumatology, University Hospital, Medical University, Sofia, Bulgaria
}

\begin{abstract}
PURPUSE: The aim of this study was to investigate the involvement of functional interleukin 10 (IL-10) and tumor necrosis factor $\alpha(\mathrm{TNF}-\alpha)$ promoter polymorphisms on the susceptibility to rheumatoid arthritis (RA) in Bulgarian population. METHODS: Genotyping for -1082A/G IL10 (rs1800896) and 308G/A TNFA (rs1800629) polymorphisms were performed using allele specific PCR and restriction fragment length RLFP-PCR assays, respectively, in 135 RA patients and 179 healthy controls. RESULTS: No association between the rs1800629 polymorphisms and RA risk in Bulgarian was established. In contrast, an association between rs1800896 and susceptibility to RA was demonstrated. There was an increase of the $I L 10-1082 \mathrm{G}$ allele $(43 \%$ vs $36.3 \%, \mathrm{p}=0.091)$ and a higher frequency of GG genotype ( $20 \%$ vs $12.8 \%$; $\mathrm{p}=0.073$ ) in cases versus controls. In logistic regression analysis the presence of high IL-10 producer genotype (-1082GG) was associated with 1.7 times higher risk of developing RA. When analyzing the influence of combined IL10/TNFA genotypes on RA appearance, we found that the carriage of both high cytokine producing genotypes of two polymorphisms (IL10 -1082GG and TNFA 308AA/GA, respectively) significantly increased the risk of developing RA with OR=4.95 $(p=0.029)$. CONCLUSIONS: Our result showed that IL10 rs1800896, but not TNFA rs1800629 gene polymorphism is associated with genetic predisposition to RA in Bulgarian population. However, IL10/TNFA interaction further influences RA susceptibility suggesting a genetic predisposition to abnormal immune regulation in this chronic inflammatory disorder.
\end{abstract}

Key words: IL-10, RA, SNP, TNF- $\alpha$

\section{INTRODUCTION}

Rheumatoid arthritis (RA) is an autoimmune rheumatic disease in which chronic inflammation is associated with pathology of the peripheral joints. RA is characterized by synovial joint inflammation and the overgrowth of synoviocytes leading to cartilage and bone destruction. CD4+ T cells are currently assumed to be the prime mediators of synovitis and the involvement of a number of cytokines in virtually all stages of rheumatoid joint inflammation, cartilage destruction and bone remodeling has been shown (1).

TNF- $\alpha$ and IL-10 have opposing roles in inflammatory responses. TNF- $\alpha$ is a key pro-

\footnotetext{
* Correspondence to: Irena Manolova, $M D, P h D$, Department of Health Care, Medical Faculty, Trakia University, Armeiska Str 11, Stara Zagora 6003, Bulgaria, e-mail: imanolova@mf.uni-sz.bg
}

inflammatory cytokine in RA pathogenesis (2). It induces tissue-specific signal transduction leading to gene expression of other cytokines such as IL-1, IL-6and L-8, involved in the pathogenic process (3). IL-10 is an immunoregulatory cytokine that is produced by almost all cells of the innate and adaptive immunity (4). Although IL-10 is associated with $\mathrm{Th} 2$ responses, it is important for $\mathrm{T}$ cell regulatory functions. There is an autoregulatory feedback loop - TNF- $\alpha$ induces the production of IL-10, which in turn reduces the TNF- $\alpha$ synthesis (5).

TNF- $\alpha$ and IL-10 can be regulated at the transcriptional level and a numbers of genetic polymorphisms in the regulatory regions of TNFA and ILIO genes are associated with changes in gene expression $(6,7)$. Among the common single nucleotide polymorphisms (SNPs), a $\mathrm{G}$ to $\mathrm{A}$ transition at position -308 in the promoter of TNFA (rs1800629) and A to G 
transition at position -1082 of IL10 gene (rs1800896) have been the most widely analyzed. Until now, several studies have examined the association of ILIO rs1800896 and TNFA rs1800629 polymorphisms with susceptibility to RA in different populations (8-10). However, the results in the literature in this respect are unconvincing. In the present study, we aimed to investigate the influence of functional ILIO -1082G/A and TNFA -308G/A promoter polymorphisms and of the combined genotypes in these loci on the susceptibility to rheumatoid arthritis (RA) in Bulgarian population.

\section{MATERIALS AND METHODS Subjects}

A total of 135 patients with RA referred to Rheumatology Clinic of St Iv Rilski University Hospital in Sofia were included in this crosssectional study and compared with 179 healthy subjects. All patients fulfilled the American College of Rheumatology classification criteria for RA (11). The group of RA patients consisted of $11(8.1 \%)$ males and $124(91.9 \%)$ females from 14 to 77 years old with a mean $( \pm$ $\mathrm{SD})$ age of $45.8 \pm 13.2$ years. The mean $( \pm \mathrm{SD})$ disease duration of RA patients was $9.5 \pm 7.9$ years (range 1-40). The sex and age distribution of the healthy controls (HC) was: $15(8.4 \%)$ males and $164(91.6 \%)$ females; aged $41.5 \pm 13.96$. years, range $20-84$ years. This study was approved by the institutional ethics committee and all subjects signed an informed consent.

\section{Genetic analysis}

Genomic DNA was extracted from peripheral whole blood using the NucleoSpin Blood L Purification kit (Macherey-Nagel, Duren, Germany) and stored at $-80^{\circ} \mathrm{C}$ until use.

Genotyping for the $-1082 \mathrm{~A} / \mathrm{G}$ SNP in IL10 (rs1800896) was performed by amplification refractory mutation system (ARMS) - PCR, using forward primer specific for $\mathrm{G}$ allele $5^{\prime}$ CCTATCCCTACTTCCCCC-3', forward primer specific for A allele 5'CCTATCCCTACTTCCCCT-3' and reverse primer generic for IL10 5'AGCAACCACTCCTCGTCGCAAC-3'. PCR amplification was performed in a GeneAmp PCR System 9700 (Applied Biosystems) as described by Stanilova et al. (12). PCR products were visualized on a $2 \%$ agarose gel stained with ethidium bromide $(0.5 \mathrm{mg} / \mathrm{ml})$.

Genotyping for the -308G/A polymorphism in the TNFA gene (rs1800629) was performed by restriction fragment length polymorphisms
(RFLP) analysis of PCR fragment amplified using the modified forward primer 5'-AGG CAA TAG GTT TTG AGG GCC AT $3^{\prime}$ and the reverse primer $5^{\prime}$-TTG GGG ACA CAC AAG CAT CAA GG 3' to create a restriction site for the NcoI enzyme as described by Manolova et al. (13). Restriction enzyme of Nco1 (Thermo Scientific) digestion with the PCR product was carried out overnight at $37^{\circ} \mathrm{C}$ and then analyzed on a 3\% agarose gel. DNA products were visualized by ethidium bromide staining. Sizes of restriction fragments were 150bp for -308 A allele and 128, 22bps for $308 \mathrm{G}$ allele.

\section{Statistical analysis.}

All data for this study were analyzed using the software SPSS version 16.0 for Windows (SPSS Inc., Chicago, IL). The differences in genotype distribution and allele frequency among cases and controls were analyzed using the $\chi 2$ test. Odd ratios with $95 \%$ confidence interval was determined to describe the strength of association by logistic regression model. The goodness of fit to Hardy-Weinberg equilibrium, calculating the expected frequencies of each genotype and comparing them with the observed values for patients and healthy controls, was performed using a $\chi^{2}$ test. In all cases, two-tailed p-values less than 0.05 were considered as significant.

\section{RESULTS}

The genotype distribution and allele frequencies of $-308 \mathrm{G} / \mathrm{A}$ in gene promoter of TNFA among cases and controls are presented in Table 1. The genotype frequencies were following Hardy-Weinberg equilibrium among cases $(p=0.723)$ and controls $(p=0.957)$. The distribution of TNFA-308 genotypes and allele frequencies were quite similar between cases and controls.

The genotype distribution and allele frequencies of $-1082 \mathrm{~A} / \mathrm{G}$ in gene promoter of ILIO among healthy controls and RA cases are presented in Table 1. Observed and expected frequencies were in Hardy-Weinberg equilibrium among cases $(\mathrm{p}=0.781)$ and controls $(\mathrm{p}=0.987)$. There was an increase of the $I L 10-1082 \mathrm{G}$ allele (43\% vs $36.3 \%)$ and decrease of the IL1O -1082 A allele (47\% vs $63.7 \%, \mathrm{p}=0.091)$. We found a higher frequency of GG genotype (20\% vs $12.8 \%$ ) and lower frequency of AA genotype in cases versus controls $(34.1 \%$ vs $40.2 \%$; $p=0.073)$. An association between RA and rs1800896 polymorphism was established under the additive model (allele $\mathrm{G}$ vs allele $\mathrm{A}$; $\mathrm{OR}=$ $1.321,95 \% \mathrm{CI}=0.94 \div 1.85, \mathrm{p}=0.091)$; the codominant model (GG vs. AA; OR $=1.837$, 
MANOLOVA I., et al.

$95 \% \mathrm{CI}=0.89 \div 3.79, \mathrm{p}=0.073)$ and the recessive model ( $\mathrm{GG}$ vs. $\mathrm{GA}+\mathrm{AA} ; \mathrm{OR}=$ $1.696,95 \% \mathrm{CI}=0.89 \div 3.25, \mathrm{p}=0.086)$. These results suppose that GG genotype could be predisposing, while AA genotype might be protective to RA susceptibility.

Table 1. Genotype and allelic frequencies of TNFA and IL10 polymorphisms in healthy controls and RA cases

\begin{tabular}{lllll}
\hline & Controls $n(\%)$ & RA $n(\%)$ & OR $(95 \%$ CI $)$ & $p$ \\
\hline TNFA $-308($ rs1800629) & & & \\
GG & $116(76.3)$ & $103(76.4 \%)$ & Reference & \\
AG & $34(22.4)$ & $31(23 \%)$ & $1.027(0.57 \div 1.85)$ & 0.925 \\
AA & $2(1.3)$ & $1(0.7 \%)$ & $0.563(0.02 \div 8.1)$ & 0.637 \\
AG+AA & $36(23.7)$ & $32(23.7 \%)$ & $1.0(0.56 \div 1.79)$ & 0.997 \\
G allele & $266(87.5 \%)$ & $237(87.8 \%)$ & Reference & \\
A allele & $38(12.5 \%)$ & $33(12.2 \%)$ & $0.98(0.58 \div 1.65)$ & 0.92 \\
IL10 -1082 (rs1800896) & & & \\
AA & $72(40.2 \%)$ & $46(34.1 \%)$ & Reference & \\
AG & $84(46.9 \%)$ & $62(45.9 \%)$ & $1.155(0.68 \div 1.95)$ & 0.567 \\
GG & $23(12.8 \%)$ & $27(20 \%)$ & $\mathbf{1 . 8 3 7}(0.89 \div 3.79)$ & $\mathbf{0 . 0 7 3}$ \\
AG+GG & $107(59.8 \%)$ & $89(65.9 \%)$ & $1.302(0.8 \div 2.13)$ & 0.265 \\
A allele & $228(63.7 \%)$ & $154(57 \%)$ & Reference & \\
G allele & $130(36.3 \%)$ & $116(43 \%)$ & $\mathbf{1 . 3 2 1}(0.94 \div 1.85)$ & $\mathbf{0 . 0 9 1}$ \\
\hline
\end{tabular}

CI, confidence interval; IL10, interleukin 10; OR, odds ratio; RA, rheumatoid arthritis, TNFA, tumor necrosis factor $\alpha$

In addition, we analyzed the effect of these two polymorphisms when combined in a genotype on RA appearance. Based on the literature data, individuals were classified as carriers of high and low producing genotypes (Table 2). We observed that the carriage of both high cytokine producing genotypes of two polymorphisms simultaneously (IL10 -1082 GG and TNFA -308 AA/GA, respectively) significantly increased the risk of developing RA with OR $=4.95$ (95\% CI: $0.94 \div 34.76 ; p=$ 0.029 .

Table 2. TNFA and IL10 genotypes and rheumatoid arthritis susceptibility

\begin{tabular}{lllll}
\hline & Controls $n(\%)$ & RA $n(\%)$ & OR $(95 \%$ CI) & $p$ \\
\hline TNFA -308 (rs1800629) & & & & \\
Low (GG) & $116(76.3)$ & $103(76.4 \%)$ & Reference & \\
High (AG/AA) & $36(23.7)$ & $32(23.7 \%)$ & $1.0(0.56 \div 1.79)$ & 0.997 \\
& & & & \\
IL10 -1082 (rs1800896) & & & & \\
Low (AG/AA) & $156(87.2 \%)$ & $108(80 \%)$ & Reference & \\
High (GG) & $23(12.8 \%)$ & $27(20 \%)$ & $\mathbf{1 . 6 9 6}(\mathbf{0 . 8 9} \div \mathbf{3 . 2 5})$ & $\mathbf{0 . 0 8 6}$ \\
& & & & \\
Combined IL10/TNFA & & & & \\
Low/Low & $104(68.4 \%)$ & $84(62.2 \%)$ & Reference & 0.658 \\
Low/High & $34(22.4 \%)$ & $24(17.8 \%)$ & $0.874(0.46-1.65)$ & $\mathbf{0 . 0 8 6}$ \\
High/Low & $12(7.9 \%)$ & $19(14.1 \%)$ & $\mathbf{1 . 9 6}(\mathbf{0 . 8 5}-\mathbf{4 . 5 8})$ & $\mathbf{4 . 9 5 2 ( \mathbf { 0 . 9 4 - 3 4 . 7 6 } )}$ \\
High/High & $2(1.3 \%)$ & $8(5.9 \%)$ & $\mathbf{0 . 0 2 9}$ \\
\hline
\end{tabular}

CI, confidence interval; IL10, interleukin 10; OR, odds ratio; RA, rheumatoid arthritis, TNFA, tumor necrosis factor $\alpha$

\section{DISCUSION}

In the present study, we found an overrepresentation of the IL10 -1082 G allele and GG genotype in Bulgarian patients with RA with borderline significance. In contrast, an apparent association was not shown between the $-308 \mathrm{G} / \mathrm{A}$ polymorphism of the TNF- $\alpha$ gene and susceptibility to RA in the studied population.

Several studies have explored the association of the TNFA SNP at locus -308 and the susceptibility to RA. Wilson et al. (7) for the first time examine the $-308 \mathrm{G} / \mathrm{A}$ SNP in relation to RA and they do not established an 
association of TNFA alleles and susceptibility to RA in Dutch ethnicity. In Spanish and French patients, no association of TNFA promoter variation at position -308 with RA susceptibility has been detected $(8,14)$. However, unlike the Caucasian, in a large cohort of Japanese patients with RA $(n=545)$ an increase of $-308 \mathrm{G}$ allele was established, which was associated with HLA-DRB $1 * 0405$ (9). Another study with a non-Caucasian population from Taiwan (15) also showed an increased frequency of the $-308 \mathrm{G}$ allele in RA patients. It could be considered that the 308G/A SNP may have a different effect on the genetic predisposition to RA development, depending on the race. In this regard, our results are in agreement with these observations, as Bulgarians belong to Caucasians.

Conflicting results were also reported regarding the possible association between IL10 -1082 A/G polymorphism and RA susceptibility in the different populations. These differences point to the importance of doing studies in the separate ethnic groups. Some works showed significant associations between ILIO -1082A/G SNP with RA susceptibility or with the development of certain clinical features (16-19), while other studies indicated that these polymorphisms did not appear to have any relevance in the disease $(20,21)$. The associations between IL-10 polymorphisms and susceptibility to RA has been recently reviewed by Lee et al. in a metaanalysis of the polymorphisms in IL-10 gene (10). This meta-analysis reveals that the $1082 \mathrm{G}$ allele in four studies in HWE showed a significant association with RA appearance with OR of 1.217. Our results are in the same direction. We found that the presence of the IL10 -1082 allele $\mathrm{G}$ in the genotype could be a risk factor for RA in Bulgarian population. In addition, individuals who carried the combined high-producing genotypes of IL10 (-1082GG) and TNFA (-308AA/AG) had a 5 times increased genetic risk for the development of RA.

With regard to IL-10 and TNF-alpha genegene interaction, Suarez et al. reported that subjects carrying the IL10 rs800896 GG and TNFA rs rs1800629 AA/AG genotypes had a 3.87-fold elevated risk of developing systemic lupus erythematosus (22), an observation similar to our results. Although the molecular mechanism is not entirely clear, the IL10 1082GG/TNFA-308A carriers may have high protein production, due to higher transcriptional activity $(6,23)$. Higher production of both pro-inflammatory TNF- $\alpha$ and anti-inflammatory IL-10, may lead to cytokine network disturbance and predisposition to autoimmunity.

In conclusion, our results demonstrate the week association of promoter polymorphism $1082 \mathrm{~A} / \mathrm{G}$ in the IL-10 gene, but not of the TNFA -308G/A polymorphic variants with genetic predisposition to rheumatoid arthritis in Bulgarian population. Moreover, subjects carrying the IL-10 rs1800896GG and TNFA 308AA/GA genotypes had a 4.95-fold increased risk of RA development.

The combined genotypes in IL10/TNFA significantly influence RA susceptibility, proving the existence of a genetic predisposition to abnormal immune regulation in this chronic inflammatory joint disease.

\section{ACKNOWLEDGMENTS}

This work was supported by Grants No:4/2009 and No:15/2014 from the Fund for Scientific and Mobile project from Faculty of Medicine at the Trakia University, Stara Zagora, Bulgaria.

\section{REFERENCES}

1. Smolen, J.J., Aletaha, D. and Redlich, K. The pathogenesis of rheumatoid arthritis: new insights from old clinical data? Nature Rev Rheumatol, 8:235-243, 2012.

2. Brennan, F.M., Maini, R.N., Feldmann, M. TNF alpha - a pivotal role in rheumatoid arthritis? $\mathrm{Br} J$ Rheumatol, 31:293-298, 1992.

3. Butler, D.M., Maini, R.N., Feldmann, M., Brennan, F.M., Modulation of proinflammatory cytokine release in rheumatoid synovial membrane cell cultures. Comparison of monoclonal anti TNF-alpha antibody with the interleukin-1 receptor antagonist. Eur Cytokine Network, 6: 225-230, 1995.

4. Moore, K.W., de Waal Malefyt, R., Coffman, R.L., O'Garra, A. Interleukin-10 and the interleukin-10 receptor. Annu Rev Immunol, 19:683-765, 2001.

5. Lio, D., Candore, G., Colombo, A., Colonna Romano, G., Gervasi, F., Marino, V., Scola, L., Caruso, C. A genetically determined high setting of TNF-alpha influences immunologic parameters of HLA-B8, DR3 positive subjects: implications for autoimmunity. Hum Immunol, 62:705-713, 2001.

6. Turner, D.M., Williams, D.M., Sankaran, D., Lazarus, M., Sinnott, P.J., Hutchinson, I.V. An investigation of polymorphism in the interleukin-10 gene promoter. Eur $J$ Immunogenet, 24:1-8, 1997. 
7. Wilson, A.G., de Vries, N., Pociot, F., di Giovine, F.S., van der Putte, L.B., Duff, G.W. An allelic polymorphism within the human tumor necrosis factor alpha promoter region is strongly associated with HLA A1, B8, and DR3 alleles. J Exp Med, 177:557-560, 1993.

8. Martínez, A., Fernández-Arquero, M., Pascual-Salcedo, D., Conejero, L., Alves, H., Balsa, A., de la Concha, E.G. Primary association of tumor necrosis factor-region genetic markers with susceptibility to rheumatoid arthritis. Arthritis Rheum, 43:1366-1370, 2000.

9. Shibue, T., Tsuchiya, N., Komata, T., Matsushita, M., Shiota, M., Ohashi, J., Wakui, M., Matsuta, K., Tokunaga, K. Tumor necrosis factor alpha 5'-flanking region, tumor necrosis factor receptor II, and HLA-DRB1 polymorphisms in Japanese patients with rheumatoid arthritis. Arthritis Rheum, 43:753-757, 2000.

10.Lee, Y.H., Bae, S.C., Choi, S.J., Ji, J.D., Song, G.G. Associations between interleukin-10 polymorphisms and susceptibility to rheumatoid arthritis: a meta-analysis. Mol Biol Rep, 39:81-87, 2012.

11. Arnett FC, Edworthy SM, Bloch DA, McShane DJ, Fries JF, Cooper NS, Healey LA, Kaplan SR, Liang MH, Luthra HS, et al. The American Rheumatism Association 1987 revised criteria for the classification of rheumatoid arthritis. Arthritis Rheum, 31:315-324, 1988.

12.Stanilova, S.A., Miteva, L.D., Karakolev, Z.T., Stefanov, C.S. Interleukin-10-1082 promoter polymorphism in association with cytokine production and sepsis susceptibility. Intensive Care Med, 32:260266, 2006.

13.Manolova, I., Ivanova, M., Stoilov, R., Rashkov, R., Stanilova, S. Association of single nucleotide polymorphism at position -308 of the tumor necrosis factor-alpha gene with ankylosing spondylitis and rheumatoid arthritis. Biotechnol Biotechnol Equip, 28:1108-1114, 2014

14.Mugnier, B., Balandraud, N., Darque, A., Roudier, C., Roudier, J., Reviron, D. Polymorphism at position -308 of the tumor necrosis factor alpha gene influences outcome of infliximab therapy in rheumatoid arthritis. Arthritis Rheum, 48:1849-1852, 2003.
15.Yen, J.H., Chen, C.J., Tsai, W.C., Lin, C.H., Ou, T.T., Wu, C.C., Liu, H.W. Tumor necrosis factor promoter polymorphisms in patients with rheumatoid arthritis in Taiwan. J Rheumatol, 28:1788-1792, 2001.

16.Paradowska-Gorycka, A., Trefler, J., Maciejewska-Stelmach, J., Lacki, J.K. Interleukin-10 gene promoter polymorphism in Polish rheumatoid arthritis patients. Int $J$ Immunogenet, 37:225-231, 2010.

17.de Paz, B, Alperi-Lopez, M., BallinaGarcia, F.J., Prado, C., Mozo, L., Gutierrez, C., Suarez, A. Interleukin 10 and tumor necrosis factor-alpha genotypes in rheumatoid arthritis-association with clinical response to glucocorticoids. $J$ Rheumatol, 37:503-511, 2010

18.Trajkov, D., Mishevska-Perchinkova, S., Karadzova-Stojanoska, A., Petlichkovski, A., Strezova, A., Spiroski, M. Association of 22 cytokine gene polymorphisms with rheumatoid arthritis in population of ethnic Macedonians. Clin Rheumatol, 28:12911300, 2009.

19.Ates, O., Hatemi, G., Hamuryudan, V., Topal-Sarikaya, A. Tumor necrosis factoralpha and interleukin-10 gene promoter polymorphisms in Turkish rheumatoid arthritis patients. Clin Rheumatol, 27:12431248, 2008.

20.Menegatti, E., Davit, A, Francica, S., Berardi, D., Rossi, D., Baldovino, S., Tovo, P.A., Sena, L.M., Roccatello, D. Genetic factors associated with rheumatoid arthritis and systemic vasculitis: evaluation of a panel of polymorphisms. Dis Markers, 27:217-223, 2009.

21.Gambhir, D., Lawrence, A., Aggarwal, A., Misra, R., Mandal, S.K., Naik, S. Association of tumor necrosis factor alpha and IL-10 promoter polymorphisms with rheumatoid arthritis in North Indian population. Rheumatol Int, 30:1211-1217, 2010.

22.Suárez A, López $\mathrm{P}$, Mozo L, Gutiérrez C. Differential effect of IL10 and TNF(alpha) genotypes on determining susceptibility to discoid and systemic lupus erythematosus Ann Rheum Dis, 64:1605-1610, 2005.

23.Kroeger, K.M., Carville, K.S., Abraham, L.J. The -308 tumor necrosis factor-alpha promoter polymorphism effects transcription. Mol Immunol, 34:391-399, 1997. 